\title{
Article \\ Determining Factors for Independent Walking in Patients Undergoing Cardiovascular Surgery: Differences between Coronary Artery Bypass Grafting, Heart Valve Surgery, and Aortic Surgery
}

\author{
Yui Sakamoto ${ }^{1}$, Yosuke Morimoto ${ }^{1,2} \mathbb{D}$, Masatoshi Hanada ${ }^{1,3} \mathbb{D}^{\mathbb{D}}$, Yudai Yano ${ }^{1,3}$, Terumitsu Sawai ${ }^{1}$, \\ Takashi Miura ${ }^{4}$, Kiyoyuki Eishi ${ }^{4}$ and Ryo Kozu $1,3, *$ D
}

Citation: Sakamoto, Y.; Morimoto, Y.; Hanada, M.; Yano, Y.; Sawai, T.; Miura, T.; Eishi, K.; Kozu, R. Determining Factors for Independent Walking in Patients Undergoing Cardiovascular Surgery: Differences between Coronary Artery Bypass Grafting, Heart Valve Surgery, and Aortic Surgery. Healthcare 2021, 9 , 1475. https://doi.org/10.3390/ healthcare 9111475

Academic Editor: Raffaele Giordano

Received: 22 September 2021

Accepted: 28 October 2021

Published: 30 October 2021

Publisher's Note: MDPI stays neutral with regard to jurisdictional claims in published maps and institutional affiliations.

Copyright: (c) 2021 by the authors. Licensee MDPI, Basel, Switzerland. This article is an open access article distributed under the terms and conditions of the Creative Commons Attribution (CC BY) license (https:// creativecommons.org/licenses/by/ $4.0 /)$.
1 Department of Cardiopulmonary Rehabilitation Science, Nagasaki University Graduate School of Biomedical Sciences, Nagasaki 852-8520, Japan; bb55317019@ms.nagasaki-u.ac.jp (Y.S.); morimoto@reha.kobegakuin.ac.jp (Y.M.); mstshnd@nagasaki-u.ac.jp (M.H.); yudai-121@nagasaki-u.ac.jp (Y.Y.); sawai@nagasaki-u.ac.jp (T.S.)

2 Department of Physical Therapy, Faculty of Rehabilitation, Kobe Gakuin University, Kobe 651-2180, Japan

3 Cardiorespiratory Division, Department of Rehabilitation Medicine, Nagasaki University Hospital, Nagasaki 852-8501, Japan

4 Department of Cardiovascular Surgery, Nagasaki University Hospital, Nagasaki 852-8501, Japan; takashirsa@nagasaki-u.ac.jp (T.M.); keishi@nagasaki-u.ac.jp (K.E.)

* Correspondence: ryokozu@nagasaki-u.ac.jp; Tel.: +81-95-819-7963

\begin{abstract}
Physical deconditioning often occurs during the acute phase after cardiovascular surgery, and unassisted walking is required to achieve independence, to manage cardiac diseases, and to prevent recurrences. This study aims to investigate the characteristics of independent walking after cardiovascular surgery. We conducted a retrospective cohort study in patients who underwent cardiovascular surgeries (total of 567 patients): 153 in the coronary artery bypass grafting (CABG) group, 312 in the heart valve surgery group, and 102 in the aortic surgery group. We evaluated the effect of each surgery group on the cardiac rehabilitation (CR) progression. The factors associated with independent walking were age, renal diseases, intensive care unit (ICU) length of stay, and post-operative respiratory complications in the CABG group. In the heart valve surgery group, the factors were New York Heart Association functional classification, renal and respiratory diseases, ICU length of stay, duration of mechanical ventilatory support, and post-operative cardiovascular and respiratory complications. In the aortic surgery group, these were ICU length of stay and acute kidney injury. The CR progression in patients who underwent aortic surgery was significantly longer than those who underwent CABG and heart valve surgery $(p<0.001)$. New intervention strategies are needed for patients with prolonged ICU stays.
\end{abstract}

Keywords: cardiac rehabilitation; thoracic surgery; cardiac surgical procedures; aortic disease; walking; mobility limitation; early ambulation; postoperative care

\section{Introduction}

Early achievement of independent walking after cardiovascular surgery plays an important role in independence in daily living, management of cardiac disease, and prevention of the recurrence of disease [1]. It is recommended that cardiac rehabilitation (CR) after cardiovascular surgery should be started in the early post-operative period, within 24-72 h after surgery [1-3]. Early CR is effective in reducing post-operative complications, in improving exercise tolerance, and in preventing delirium [1,4-8]. In addition, independent walking is targeted to be achieved within 4-7 days after surgery, and continued exercise training, mainly aerobic exercise such as walking, is effective in reducing rehospitalization and coronary risk factors and in enhancing quality of life [1,9-15]. However, 
patients who have undergone cardiovascular surgery are often subjected to prolonged immobilization $[16,17]$. Surgery and subsequent prolonged bed rest can lead to rapid deterioration of the musculoskeletal system [18,19]. Monteleone et al. [20] reported that more than half of patients after cardiothoracic surgery had physical dysfunction and that $25.5 \%$ of patients were unable to walk independently at discharge. Independent walking after cardiovascular surgery has received attention as an important acquired behavior in previous studies $[16,17,20]$.

Saitoh et al. [16] showed that post-operative renal function and fluid balance were independent factors that determine walking ability in the early post-operative period in patients undergoing coronary artery bypass grafting $(\mathrm{CABG})$ and valvular heart disease. Kato et al. [17] identified age, serum albumin levels, operative time, and post-operative atrial fibrillation as independent predictors of walking delay during $C R$ in patients who underwent heart valve surgery. After aortic surgery, bed rest was likely to be a priority, and there were significantly fewer prior studies on CR than in other cardiac fields [12] and no prior studies on the determinants of independent walking. Additionally, post-aortic surgery is a major indication for CR $[1,8,12,13]$, as the number of such surgeries is increasing every year [21] and post-operative survival rates are also rising [22]. Aortic surgery, which is highly invasive, is expected to cause significant impairment in walking independence in the early post-operative period due to complications and other deconditioning factors [12,23-25].

The current limitation in the literature is that studies examining the factors that determine independent walking are limited to the type of surgery. Increased knowledge of the determinants of independent walking may be useful for appropriate post-operative $\mathrm{CR}$ and resource management, and early independent walking may contribute to shorter hospital stays and lower medical costs [4-8]. Therefore, the aims of this study were (1) to investigate the characteristics of delayed achievement of independent walking after different types of cardiovascular surgery and (2) to evaluate the CR progression in different surgical techniques.

\section{Materials and Methods}

\subsection{Study Design}

This retrospective cohort study was conducted at a single center. We enrolled patients who underwent CR after cardiovascular surgery at Nagasaki University Hospital between June 2013 and December 2016. This study was approved by the Nagasaki University Hospital Clinical Research Ethical Committee (reference number 15061113). Informed consent was obtained in the form of an opt-out option on the website; those who were not interested were excluded.

\subsection{Subjects}

We included patients who underwent CR after all cardiovascular surgeries, except surgery for abdominal aortic aneurysms and lower extremity bypass surgery. Of them, the exclusion criteria for patients in this study were being under 18 years of age at the time of surgery, having underwent combined cardiac and aortic surgery, repeated surgery or surgery with general anesthesia within one-month, post-operative stroke, and death in the hospital after surgery. Patients who were unable to walk before or after cardiovascular surgery, even with the use of a walking aid (e.g., cane, crutches, walker), were also excluded.

\subsection{Data Collection}

Using an electronic medical record system, we obtained data on the demographics of the patients from hospital admission to discharge. The pre-operative data were age, sex, body mass index (BMI), New York Heart Association (NYHA) functional classification, cardiac function (left ventricular ejection fraction: LVEF, the ratio of mitral peak velocity of early filling to early diastolic mitral annular velocity: E/e'), pre-operative diagnosis, and comorbidities. Comorbidities, including hypertension [26], diabetes [27], renal disease 
(chronic kidney disease), respiratory disease (chronic obstructive pulmonary disease, interstitial pneumonia, and bronchial asthma, and pneumoconiosis), and central nervous system disease (stroke and brain tumor), were diagnosed by the cardiovascular surgeon.

The intra-operative data included operative time, amount of bleeding, aortic cross clumping time, cardiopulmonary bypass time, and the required number of emergency surgeries. The post-operative data included intensive care unit (ICU) length of stay, duration of mechanical ventilation, complications, progression of $\mathrm{CR}$, and the overall length of stay in the hospital. The duration of mechanical ventilation was defined as the time from immediately after the surgery to successful extubation (no re-intubation within 24 hours). Post-operative complications, either as a newly developed or an exacerbated comorbidity disease, until the completion of independent walking for up to one month, were diagnosed by the cardiovascular surgeon or intensivist. These were defined as follows: cardiovascular complications (ischemic heart disease, infective endocarditis, pericardial effusion, bradyarrhythmia, supraventricular arrhythmia, and ventricular arrhythmia) treated with more than local anesthesia such as cardiac catheterization, replacement of existing drains, placement or puncture of new drains into the pericardial cavity, pacemaker implantation, acute kidney injury (AKI) [28], delirium [29], and respiratory complications (upper and lower airway infections, pneumonia, respiratory failure, atelectasis, and pneumothorax) [30,31]. For respiratory infections, we followed the criteria described by Canet [30]. Respiratory failure was defined as being on mechanical ventilation for $>48$ hours after the surgery [31].

\subsection{Early Post-Operative CR Program}

The CR program was based on the Japanese Circulation Society's early post-operative CR guidelines as follows: (1) initiated on the post-operative day 1 , number of sessions at 1-2 times/day, the frequency at 6 times/week daily, and continued until discharge; (2) carried out under the supervision of a physical therapist; and (3) consisted of passive and active limb exercises, respiratory physical therapy, and early mobilization (sitting on the edge of a bed, standing, walking, and exercise training) [1].

Progression during early post-operative $\mathrm{CR}$ was analyzed using the following criteria: the initiation of mobilization, including sitting on the edge of the bed and standing at the bedside; initial walking; and the completion of $200 \mathrm{~m}$ walking. In accordance with the Japanese Circulation Society's early post-operative CR guidelines, we defined $200 \mathrm{~m}$ walking as achieving independent walking and as the goal of early post-operative CR [1].

\subsection{Statistical Analyses}

The distribution of the data was analyzed using the Shapiro-Wilk test. When the data were not normally distributed, a non-parametric test was used. Demographics and perioperative data of patients, for each type of surgery, were compared by one-way analysis of variance; if the results were significant, multiple comparisons with Bonferroni correction were performed. Simple and multiple linear regression analyses were performed to assess the factors related to independent walking for each type of surgery. The variables identified as significant $(p<0.05)$ on single linear regression analysis were used as independent variables for multiple regression analyses, considering multicollinearity. Pearson productmoment correlation was used to examine the relationship between the ICU length of stay and progression of CR. Values are expressed as median (interquartile range) or number $(\%)$. The level of statistical significance was set at $p<0.05$. All statistical analyses were performed using JMP®15.0 (SAS Institute Inc., Cary, NC, USA).

\section{Results}

The study cohort initially consisted of 763 consecutive patients who underwent cardiovascular surgery during the study period. Of them, patients who met the exclusion criteria were as follows. Patients who underwent combined cardiac and aortic surgery $(n=80)$, who could not walk after surgery until discharge $(n=32)$, who died during hospitalization $(n=23)$, who could not walk before surgery $(n=17)$, who had post-operative stroke $(n=17)$, 
who were under 18 years of age $(n=10)$, who underwent repeat cardiovascular surgery within one month $(n=10)$, and who underwent other surgeries within one month $(n=7)$. Consequently, a total of 567 patients were included in the study.

\subsection{Demographics of Patients}

The peri-operative demographics of patients are shown in Table 1 . The demographics were classified as follows: CABG ( $n=153$ (on-pump, $n=80$; off-pump, $n=73$ )), heart valve surgery ( $n=312$ (minimally invasive cardiac surgery, $n=120)$ ), and aortic surgery $(n=102)$. The pre-operative diagnosis for CABG was angina pectoris $(n=133)$, asymptomatic myocardial ischemia $(n=12)$, and myocardial infarction $(n=8)$. The pre-operative diagnosis for heart valve surgery was valvular insufficiency $(n=165)$, valvular stenosis $(n=84)$, combination of valvular insufficiency and stenosis $(n=23)$, combined valvular disease $(n=18)$, infectious endocarditis $(n=7)$, and others, including chronic heart failure and intra-cardiac thrombosis $(n=15)$. The pre-operative diagnosis for aortic surgery was aortic aneurysm $(n=34)$, dissociative aortic aneurysm $(n=32)$, acute aortic dissection $(n=19)$, aortic aneurysm rupture $(n=5)$, and others, including infection of artificial blood vessels and aortic root disease $(n=12)$.

Table 1. Demographics and peri-operative data of patients.

\begin{tabular}{|c|c|c|c|c|c|c|c|}
\hline \multirow{2}{*}{$\begin{array}{l}\text { Variables } \\
\text { Age, year }\end{array}$} & \multicolumn{2}{|l|}{$\begin{array}{l}\text { CABG Group } \\
\quad(n=153)\end{array}$} & \multicolumn{2}{|c|}{$\begin{array}{l}\text { Heart Valve Surgery Group } \\
\qquad(n=312)\end{array}$} & \multicolumn{2}{|c|}{$\begin{array}{l}\text { Aortic Surgery Group } \\
\qquad(n=102)\end{array}$} & \multirow{2}{*}{$\frac{p \text { Value }^{\mathrm{a}}}{0.205}$} \\
\hline & $69.0[63.0-77.5]$ & & $70.0[60.0-77.0]$ & & $69.0[58.0-78.0]$ & & \\
\hline Male, n (\%) & $115(75.2)$ & & $155(49.7)$ & $t+t$ & $67(65.7)$ & $\ddagger$ & $<0.001$ \\
\hline $\mathrm{BMI}, \mathrm{kg} / \mathrm{m}^{2}$ & 23.8 [21.6-26.5] & $*$ & 22.4 [20.2-24.7] & $t+t$ & 23.2 [20.7-24.8] & & $<0.001$ \\
\hline \multicolumn{8}{|l|}{$\begin{array}{l}\text { NYHA functional } \\
\text { classification, n (\%) }\end{array}$} \\
\hline I/II/III/IV/Unknown & $\begin{array}{c}43(28.1) / 45(29.4) / 39 \\
(25.5) / 24(15.7) / 2 \\
(1.3)\end{array}$ & & $\begin{array}{c}72(23.1) / 109 \\
(34.9) / 93(29.8) / 36 \\
(11.5) / 2(0.6)\end{array}$ & & $\begin{array}{l}53(52.0) / 20(19.6) / 10 \\
(9.8) / 4(3.9) / 15(14.7)\end{array}$ & $\ddagger \ddagger \ddagger$ & $<0.001$ \\
\hline LVEF, \% & $65.5[54.8-72.3]$ & & $66.0[58.0-72.0]$ & & $66.0[59.0-70.0]$ & & 0.700 \\
\hline $\mathrm{E} / \mathrm{e}^{\prime}, \mathrm{cm} / \mathrm{s}$ & $10.6[8.0-13.7]$ & & $14.3[10.6-20.4]$ & t+t & $9.0[7.6-12.5]$ & 㧊 & $<0.001$ \\
\hline \multicolumn{8}{|l|}{ Comorbidities, n (\%) } \\
\hline Hypertension & $90(58.8)$ & & $133(42.6)$ & tt & $64(62.7)$ & 拉 & $<0.001$ \\
\hline Diabetes & $62(40.5)$ & $* * *$ & $55(17.6)$ & t+t & $7(6.9)$ & $\ddagger$ & $<0.001$ \\
\hline Renal disease & $28(18.3)$ & & $66(21.2)$ & & $11(10.8)$ & & 0.058 \\
\hline \multirow{2}{*}{$\begin{array}{c}\text { Respiratory disease } \\
\text { Central nervous system } \\
\text { disease }\end{array}$} & $25(16.3)$ & & $59(18.9)$ & & $14(13.7)$ & & 0.471 \\
\hline & $28(18.3)$ & & $32(10.3)$ & + & $10(9.8)$ & & 0.040 \\
\hline Operative time, $\min$ & $304.0[248.5-340.5]$ & $* * *$ & 257.5 [221.5-294.8] & $t+t$ & $381.0[321.8-457.0]$ & 㧊 & $<0.001$ \\
\hline \multirow{2}{*}{$\begin{array}{l}\text { Amount of bleeding, } \mathrm{mL} \\
\text { Aortic cross clumping } \\
\text { time, } \mathrm{min}\end{array}$} & $1010.0[500.0-1649.5]$ & $* * *$ & $1230.0[545.5-1800.0]$ & & $2290.0[980.0-3495.0]$ & 㧊 & $<0.001$ \\
\hline & $0.0[0-74.5]$ & $* * *$ & 80.0 [68.0-108.0] & $+t+$ & 135.0 [102.3-169.5] & 执 & $<0.001$ \\
\hline $\begin{array}{c}\text { Cardiopulmonary bypass } \\
\text { time, } \min \end{array}$ & 72.0 [0-119] & $* * *$ & 135.0 [111.0-166.0] & t+t & 207.0 [161.8-246.5] & 执 & $<0.001$ \\
\hline \multirow{2}{*}{$\begin{array}{l}\text { Emergency surgery, n (\%) } \\
\text { Length of intensive care } \\
\text { unit stay, day }\end{array}$} & $22(14.4)$ & $* * *$ & $5(1.6)$ & t+t & $50(49.0)$ & 㧊 & $<0.001$ \\
\hline & 1.0 [1.0-2.0] & $* * *$ & $1.0[0-2.0]$ & & $2.0[1.0-5.0]$ & $\ddagger \ddagger \ddagger$ & $<0.001$ \\
\hline $\begin{array}{l}\text { Duration of mechanical } \\
\text { ventilatory support, hour }\end{array}$ & 11.8 [8.6-17.9] & $* * *$ & 9.6 [7.4-14.9] & t+t & 18.8 [11.8-25.1] & 㧊 & $<0.001$ \\
\hline $\begin{array}{l}\text { Post-operative } \\
\text { complications, } \mathrm{n}(\%)\end{array}$ & $62(40.52)$ & $* * *$ & $116(37.2)$ & & $71(69.6)$ & 㧊 & $<0.001$ \\
\hline Cardiovascular & $13(8.6)$ & & $40(12.8)$ & & $10(9.8)$ & & 0.372 \\
\hline Acute kidney injury & $2(1.3)$ & & $9(2.9)$ & & $7(6.9)$ & & 0.052 \\
\hline Delirium & $11(7.2)$ & & $20(6.4)$ & & $13(12.7)$ & & 0.135 \\
\hline Respiratory & $50(32.7)$ & $* * *$ & $79(25.3)$ & & $67(65.7)$ & 㧊 & $<0.001$ \\
\hline \multicolumn{8}{|l|}{$\begin{array}{c}\text { Cardiac rehabilitation } \\
\text { progress }\end{array}$} \\
\hline $\begin{array}{l}\text { Sitting on the edge of a } \\
\text { bed, day }\end{array}$ & $1.0[1.0-2.0]$ & $* * *$ & $1.0[1.0-1.0]$ & & $2.0[1.0-3.0]$ & 㧊 & $<0.001$ \\
\hline Standing, day & $1.0[1.0-2.0]$ & $* * *$ & $1.0[1.0-2.0]$ & & $2.0[1.0-4.0]$ & 㧊 & $<0.001$ \\
\hline Initial walking, day & $3.0[2.0-4.0]$ & $* * *$ & $2.0[2.0-4.0]$ & t+ & $4.0[3.0-6.3]$ & 㧊 & $<0.001$ \\
\hline
\end{tabular}


Table 1. Cont.

\begin{tabular}{|c|c|c|c|c|c|c|c|}
\hline \multirow{2}{*}{$\begin{array}{l}\text { Variables } \\
\text { Independent } \\
\text { walking, day }\end{array}$} & \multicolumn{2}{|c|}{$\begin{array}{l}\text { CABG Group } \\
\quad(n=153)\end{array}$} & \multicolumn{2}{|c|}{$\begin{array}{l}\text { Heart Valve Surgery Group } \\
\qquad(n=312)\end{array}$} & \multicolumn{2}{|c|}{$\begin{array}{l}\text { Aortic Surgery Group } \\
(n=102)\end{array}$} & \multirow{2}{*}{$\begin{array}{c}p \text { Value }^{\mathrm{a}} \\
\quad<0.001\end{array}$} \\
\hline & $5.0[4.0-7.0]$ & $* * *$ & $5.0[3.0-6.0]$ & + & $8.0[5.0-12.0]$ & ‡ł‡ & \\
\hline $\begin{array}{l}\text { Length of stay in } \\
\text { hospital, day }\end{array}$ & 18.0 [15.0-21.0] & $* * *$ & 18.0 [15.0-24.0] & & 23.5 [19.0-30.0] & 㧊 & $<0.001$ \\
\hline
\end{tabular}

Abbreviation: BMI: Body Mass Index, CABG: Coronary Artery Bypass Graft, E/e': The ratio of mitral peak velocity of early filling to early diastolic mitral annular velocity, LVEF: Left Ventricle Ejection Fraction, NYHA: New York Heart Association. Notes: Data are presented as median [quartile] and number (\%). ${ }^{a} p$ Value by one-way analysis of variance. Multiple comparisons test: CABG (vs. Aortic surgery): ${ }^{*} p<0.05,{ }^{* * *} p<0.001$, Valve replacement and angioplasty (vs. CABG): $+p<0.05,+\dagger p<0.01$, $++\dagger p<0.001$, Aortic surgery (vs. Valve

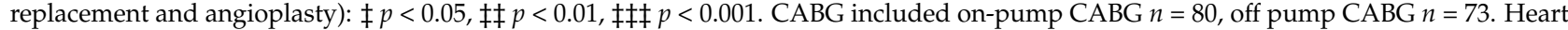
valve surgery included cardiovascular surgery $n=192$, minimally invasive cardiac surgery $n=120$.

\subsection{Post-Operative Progresssion of CR}

The post-operative progression during CR, according to the type of surgery, is shown in Table 1. The initiation of sitting on the edge of a bed and standing at the bedside was not significantly different between $C A B G$ and heart valve surgery patients but was significantly achieved later in those with aortic surgery $(p<0.001)$. Initial walking and independent walking were significantly delayed in heart valve surgery, CABG, and aortic surgery patients $(p<0.05)$, in that given order.

\subsection{Factors Related to Post-Operative Independent Walking}

The results of the determining factors related to independent walking, for each surgery, on simple linear regression analysis and multiple linear regression analysis are shown in Tables 2 and 3, respectively. As a result of multiple linear regression analysis, the determining factors in the CABG group were age, renal disease as a comorbidity, ICU length of stay, and post-operative respiratory complications. In the heart valve surgery group, the determining factors were NYHA functional classification, renal disease and respiratory disease as comorbidities, ICU length of stay, duration of mechanical ventilatory support, and post-operative cardiovascular and respiratory complications. In the aortic surgery group, the only determining factors were the ICU length of stay and post-operative AKI. ICU length of stay was a determining factor for all three types of surgery.

Table 2. Single liner regression analysis for determining factors for achieving independent walking.

\begin{tabular}{|c|c|c|c|c|c|c|}
\hline & $\begin{array}{l}\text { CABG Group } \\
\quad(n=153)\end{array}$ & & $\begin{array}{c}\text { Heart Valve } \\
\text { Surgery Group } \\
\quad(n=312)\end{array}$ & & $\begin{array}{l}\text { Aortic Surgery Group } \\
\qquad(n=102)\end{array}$ & \\
\hline & $\beta[95 \% \mathrm{CI}]$ & $p$ Value & $\beta[95 \% \mathrm{CI}]$ & $p$ Value & $\beta[95 \% \mathrm{CI}]$ & $p$ Value \\
\hline Age, year & 0.109 [0.046-0.173] & $<0.001$ & 0.074 [0.019-0.128] & 0.009 & $0.023[-0.101-0.147]$ & 0.714 \\
\hline Male (presence) & $-0.138[-0.941-0.665]$ & 0.735 & $-0.453[-1.202-0.296]$ & 0.235 & $-0.021[-1.751-1.709]$ & 0.981 \\
\hline BMI, $\mathrm{kg} / \mathrm{m}^{2}$ & $-0.217[-0.414-0.020]$ & 0.031 & $-0.209[-0.431-0.012]$ & 0.064 & $-0.314[-0.170-0.797]$ & 0.201 \\
\hline $\begin{array}{l}\text { NYHA functional } \\
\text { classification, class }\end{array}$ & $0.501[-0.158-1.160]$ & 0.135 & 1.467 [0.692-2.243] & $<0.001$ & $-0.184[-1.933-1.565]$ & 0.835 \\
\hline LVEF, \% & $-0.021[-0.079-0.038]$ & 0.486 & $-0.075[-0.131-0.019]$ & 0.008 & $0.098[-0.082-0.278]$ & 0.098 \\
\hline $\mathrm{E} / \mathrm{e}^{\prime}, \mathrm{cm} / \mathrm{s}$ & $0.205[0.039-0.370]$ & 0.016 & $0.063[-0.019-0.144]$ & 0.130 & $0.301[-0.149-0.752]$ & 0.187 \\
\hline Comorbidity (presence) & & & & & & \\
\hline Hypertension & $-0.260[-0.964-0.444]$ & 0.466 & $-0.465[-1.222-0.291]$ & 0.227 & $0.791[-0.889-2.471]$ & 0.352 \\
\hline Diabetes & $0.201[-0.506-0.907]$ & 0.576 & $0.044[-0.941-1.028]$ & 0.930 & $-1.215[-4.425-1.995]$ & 0.454 \\
\hline Renal disease & $1.615[0.756-2.475]$ & $<0.001$ & $2.279[1.396-3.161]$ & $<0.001$ & $2.433[-0.146-5.012]$ & 0.064 \\
\hline Respiratory disease & 1.014 [0.089-1.939] & 0.032 & 1.365 [0.419-2.310] & 0.001 & $1.035[-1.397-3.468]$ & 0.400 \\
\hline $\begin{array}{l}\text { Central nervous } \\
\text { system disease }\end{array}$ & 0.719 [0.171-1.610] & 0.113 & $0.086[-1.151-1.322]$ & 0.892 & $1.321[-1.403-4.046]$ & 0.334 \\
\hline Operative time, $\min$ & $0.006[-0.004-0.016]$ & 0.215 & $0.012[0.001-0.022]$ & 0.025 & $0.031[0.020-0.041]$ & $<0.001$ \\
\hline Amount of bleeding, $\mathrm{mL}$ & $0.001[8.377-0.002]$ & 0.048 & $0.001[-0.001-0.001]$ & 0.584 & $0.001[0.001-0.001]$ & $<0.001$ \\
\hline $\begin{array}{l}\text { Aortic cross clumping } \\
\text { time, } \min \end{array}$ & $0.008[-0.009-0.024]$ & 0.364 & $0.020[-0.002-0.042]$ & 0.074 & 0.036 [0.007-0.065] & 0.017 \\
\hline $\begin{array}{l}\text { Cardiopulmonary } \\
\text { bypass time, min }\end{array}$ & $0.001[-0.003-0.019]$ & 0.158 & $0.005[-0.008-0.018]$ & 0.436 & 0.024 [0.005-0.043] & 0.014 \\
\hline
\end{tabular}


Table 2. Cont.

\begin{tabular}{|c|c|c|c|c|c|c|}
\hline & $\begin{array}{l}\text { CABG Group } \\
\quad(n=153)\end{array}$ & & $\begin{array}{l}\text { Heart Valve } \\
\text { Surgery Group } \\
\quad(n=312)\end{array}$ & & $\begin{array}{l}\text { Aortic Surgery Group } \\
\qquad(n=102)\end{array}$ & \\
\hline & $\beta[95 \% \mathrm{CI}]$ & $p$ Value & $\beta[95 \% \mathrm{CI}]$ & $p$ Value & $\beta[95 \% \mathrm{CI}]$ & $p$ Value \\
\hline $\begin{array}{c}\text { Emergency surgery } \\
\text { (presence) }\end{array}$ & 2.069 [1.137-3.000] & $<0.001$ & $0.739[-2.247-3.725]$ & 0.627 & $1.490[-0.118-3.098]$ & 0.069 \\
\hline $\begin{array}{c}\text { Length of intensive care } \\
\text { unit stay, day }\end{array}$ & 1.428 [1.136-1.713] & $<0.001$ & 2.137 [1.958-2.316] & $<0.001$ & $2.261[1.801-2.721]$ & $<0.001$ \\
\hline $\begin{array}{l}\text { Duration of mechanical } \\
\text { ventilatory support, hour } \\
\text { Post-operative } \\
\text { complications (presence) }\end{array}$ & $0.047[0.018-0.076]$ & 0.002 & 0.152 [0.135-0.168] & $<0.001$ & $0.182[0.071-0.293]$ & 0.001 \\
\hline Cardiovascular & $1.318[0.087-2.548]$ & 0.036 & 2.698 [1.617-3.779] & $<0.001$ & $3.375[0.722-6.028]$ & 0.013 \\
\hline Acute kidney injury & $1.265[-1.784-4.314]$ & 0.414 & $2.282[0.055-4.508]$ & 0.045 & 7.534 [4.688-10.381] & $<0.001$ \\
\hline Delirium & 1.394 [0.069-2.719] & 0.039 & 2.834 [1.335-4.332] & $<0.001$ & $3.154[0.796-5.513]$ & 0.009 \\
\hline Respiratory & 2.107 [1.449-2.765] & $<0.001$ & 2.785 [1.981-3.590] & $<0.001$ & $2.671[1.037-4.304]$ & 0.002 \\
\hline \multicolumn{7}{|l|}{$\begin{array}{l}\text { Types of surgery } \\
\text { (presence) }\end{array}$} \\
\hline Off-pump CABG & $-0.507[-1.198-0.183]$ & 0.148 & & & & \\
\hline MICS & & & $\begin{array}{c}-1.338 \\
{[-2.094--0.581]}\end{array}$ & $<0.001$ & & \\
\hline
\end{tabular}

Abbreviation: BMI: Body Mass Index, CABG: Coronary Artery Bypass Graft, E/e': The ratio of mitral peak velocity of early filling to early diastolic mitral annular velocity, LVEF: Left Ventricle Ejection Fraction, MICS: minimally invasive cardiac surgery, NYHA: New York Heart Association. Notes: CABG included on-pump CABG $n=80$, off-pump CABG $n=73$. Heart valve surgery included MICS $n=120$.

Table 3. Multiple liner regression analysis of determining factors for achieving of independent walking.

\begin{tabular}{|c|c|c|c|c|c|c|}
\hline & \multicolumn{2}{|c|}{$\begin{array}{l}\text { CABG Group } \\
(n=153)\end{array}$} & \multicolumn{2}{|c|}{$\begin{array}{l}\text { Heart Valve Surgery Group } \\
\qquad(n=312)\end{array}$} & \multicolumn{2}{|c|}{$\begin{array}{l}\text { Aortic Surgery Group } \\
\qquad(n=102)\end{array}$} \\
\hline & \multicolumn{2}{|c|}{$\mathrm{R}^{2}=0.499, p<0.001$} & \multicolumn{2}{|c|}{$\mathrm{R}^{2}=0.698, p<0.001$} & \multicolumn{2}{|c|}{$\mathrm{R}^{2}=0.606, p<0.001$} \\
\hline & $\beta[95 \% \mathrm{CI}]$ & $p$ Value & $\beta[95 \% \mathrm{CI}]$ & $p$ Value & $\beta[95 \% \mathrm{CI}]$ & $p$ Value \\
\hline Age, year & $0.057[0.002-0.111]$ & 0.041 & $-0.005[-0.039-0.029]$ & 0.776 & & \\
\hline BMI, $\mathrm{kg} / \mathrm{m}^{2}$ & $-0.064[-0.221-0.093]$ & 0.421 & & & & \\
\hline $\begin{array}{l}\text { NYHA functional } \\
\text { classification, class }\end{array}$ & & & $0.803[0.355-1.251]$ & $<0.001$ & & \\
\hline $\mathrm{E} / \mathrm{e}^{\prime}, \mathrm{cm} / \mathrm{s}$ & $0.119[-0.015-0.252]$ & 0.082 & & & & \\
\hline Comorbidity (presence) & & & & & & \\
\hline Renal disease & 0.898 [0.177-1.619] & 0.015 & 0.688 [0.139-1.238] & 0.014 & & \\
\hline Respiratory disease & $0.327[-0.367-1.021]$ & 0.354 & $0.547[0.013-1.080]$ & 0.045 & & \\
\hline Operative time, $\min$ & & & $-0.001[-0.007-0.006]$ & 0.913 & $0.016[-0.001-0.032]$ & 0.061 \\
\hline Amount of bleeding, $\mathrm{mL}$ & $0.001[-0.001-0.001]$ & 0.234 & & & $0.022[-0.037-0.081]$ & 0.458 \\
\hline $\begin{array}{l}\text { Aortic cross clumping } \\
\text { time, min }\end{array}$ & & & & & $-0.008[-0.050-0.034]$ & 0.712 \\
\hline $\begin{array}{l}\text { Cardiopulmonary } \\
\text { bypass time, min }\end{array}$ & & & & & $-0.006[-0.034-0.023]$ & 0.681 \\
\hline $\begin{array}{c}\text { Emergency surgery } \\
\text { (presence) }\end{array}$ & $0.942[-0.160-2.043]$ & 0.093 & & & & \\
\hline $\begin{array}{c}\text { Length of intensive care } \\
\text { unit stay, day }\end{array}$ & 0.863 [0.499-1.226] & $<0.001$ & 1.326 [1.003-1.649] & $<0.001$ & 1.503 [0.947-2.058] & $<0.001$ \\
\hline $\begin{array}{l}\text { Duration of mechanical } \\
\text { ventilatory support, hour } \\
\text { Post-operative } \\
\text { complications (presence) }\end{array}$ & $0.010[-0.014-0.033]$ & 0.426 & $0.054[0.031-0.078]$ & $<0.001$ & $-0.010[-0.122-0.101]$ & 0.858 \\
\hline $\begin{array}{l}\text { Cardiovascular } \\
\text { complications }\end{array}$ & $0.663[-0.385-1.711]$ & 0.213 & 0.835 [-0.192-1.479] & 0.011 & $1.682[-0.226-3.589]$ & 0.083 \\
\hline Acute kidney injury & & & $-0.453[-1.761-0.855]$ & 0.496 & $3.062[0.451-5.673]$ & 0.022 \\
\hline Delirium & $0.155[-0.903-1.214]$ & 0.772 & $0.588[-0.317-1.494]$ & 0.202 & $1.052[-0.770-2.874]$ & 0.254 \\
\hline $\begin{array}{l}\text { Respiratory } \\
\text { complications } \\
\text { Types of surgery } \\
\text { (presence) }\end{array}$ & 0.911 [0.269-1.555] & 0.006 & 0.841 [0.303-1.378] & 0.002 & $0.187[-1.060-1.434]$ & 0.767 \\
\hline
\end{tabular}

Abbreviation: BMI: Body Mass Index, CABG: Coronary Artery Bypass Graft, E/e': The ratio of mitral peak velocity of early filling to early diastolic mitral annular velocity, MICS: minimally invasive cardiac surgery, NYHA: New York Heart Association. Notes: CABG included on-pump CABG $\mathrm{n}=80$, off pump CABG $\mathrm{n}=73$. Heart valve surgery included MICS $\mathrm{n}=120$. 


\subsection{Relationships between ICU Length of Stay and Progression of CR in the Aortic Surgery Group}

The relationship between the ICU length of stay and the progression of $C R$ in patients who underwent aortic surgery is shown in Figure 1. The ICU length of stay was positively correlated with progression during CR (initial walking $(r=0.779, p<0.001)$, independent walking $(r=0.700, p<0.001)$, standing $(r=0.693, p<0.001)$, and sitting on the edge of a bed $(r=0.645, p<0.001))$.
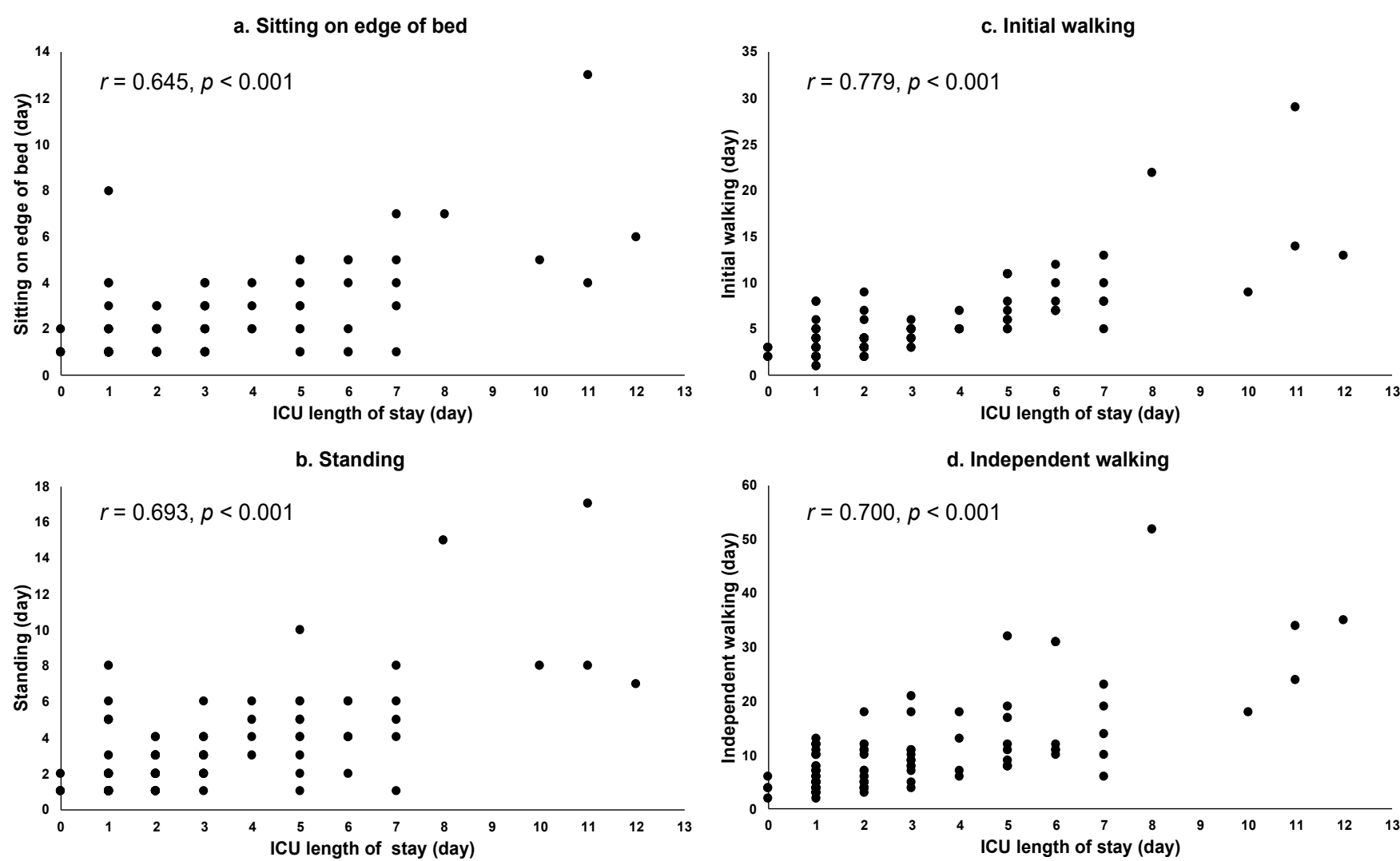

Figure 1. Relationships between ICU length of stay and CR in aortic surgery group. Abbreviation: intensive care unit: ICU. Notes: (a) correlation between ICU length of stay and achieving sitting on the edge of a bed, (b) correlation between ICU length of stay and achieving standing, (c) correlation between ICU length of stay and achieving initial walking, and (d) correlation between ICU length of stay and achieving independent walking.

\section{Discussion}

The main findings of the present study were as follows: (1) the ICU length of stay was a common determining factor for the influence of independent walking in all three groups; (2) post-operative respiratory complications were a common determining factor for independent walking in the CABG and valve groups; (3) post-operative AKI was a determining factor for independent walking only in the aortic surgery group; and (4) progression of CR in patients with aortic surgery was significantly later than it was in the CABG and heart valve surgery patients. These results indicate that there are both common and specific factors associated with post-operative independent walking in each of the three surgical groups. To our knowledge, this is the first study to examine in detail the determining factors for independent walking according to different types of cardiovascular surgery.

According to the results of our study, the ICU length of stay was an important factor in determining independent walking after the cardiovascular surgery, regardless of the surgical type. A previous study reported that $47 \%$ of ICU patients could not be mobilized because of respiratory and hemodynamic instability, problems with staffing, and a busy ICU environment [32]. In this study, the ICU length of stay was correlated with both independent walking and the progress of early post-operative CR. These findings suggest 
that patients in the ICU for a long period were not only in poor post-operative condition but also do not walk and spend less time in mobility because of the ICU-specific environment.

Factors determining independent walking were extracted as post-operative respiratory complications in the CABG group and the valve surgery group. Post-operative respiratory complications in the aortic surgery group were not a determining factor for independent walking but had the highest incidence $(65 \%)$ among the three surgical groups. Canet et al. [30], which was referred to for the definition of post-operative respiratory complications in this study, reported a post-operative incidence of $39.6 \%$, which was similar to the incidence in the CABG and valvular surgery groups of this study. Post-operative respiratory complications have been reported to influence increased mortality, prolonged hospital stay, and progression of CR [30,33]. The higher incidence of post-operative respiratory complications in the aortic surgery group compared with the other two surgery groups suggests that more patients were affected by their post-operative general condition than in the other two surgery groups, which may have contributed to the overall delay in CR for aortic surgery compared with the other two surgery groups.

AKI was extracted as an independent factor determining independent walking in the aortic surgery group. In previous studies, cardiopulmonary bypass time is independently associated with an increased risk of post-operative AKI in patients after the aortic surgery [23]. Similar to earlier studies, we also observed a longer cardiopulmonary bypass time in patients with aortic surgery, compared with the CABG and heart valve surgery patients. Post-operative AKI has also been reported to cause a delay in CR due to a longer critical care period after cardiac surgery [16]. Specifically, the management of AKI requires early dialysis or continuous hemodiafiltration for kidney damage and various complications. These may have a direct impact on the early progression of $\mathrm{CR}$, including independent walking.

In previous studies [16,17], pre- and intra-operative factors were associated with achieving independent walking in patients who underwent CABG and heart valve surgery; these findings, with regard to $C A B G$ and heart valve surgery, were consistent with the results of our study. However, in the aortic surgery group, post-operative factors such as length of ICU stay and post-operative AKI, but not pre-operative factors, were associated with independent walking. In addition, in the aortic surgery group, pre-operative factors as determinants of independent walking were not extracted as a significant factor in the single regression analysis. These findings indicate that the post-operative factors may play an important role in walking during $\mathrm{CR}$ after aortic surgery. The post-operative factors in the aortic surgery group were the significantly longer operative time and greater amount of bleeding, an increase in the number of emergency surgeries, the longer duration of mechanical ventilatory support, and a higher incidence of complications compared with the other two surgery groups. This indicates that aortic surgery is more invasive than the other two surgical groups, which may be the cause of the longer post-operative functional recovery.

In this study, the progression of CR in the enrolled patients was in accordance with that observed in previous studies $[1,16,17]$. Compared with the other two surgical groups, $\mathrm{CR}$ progression in the aortic surgery group was significantly delayed from sitting on the edge of a bed to independent walking. The length of hospital stay was also the longest among the three surgical groups. These results suggest that aortic surgery, compared with other cardiac surgeries, is more likely to interfere with post-operative recovery. As an approach to early recovery of physical functions, it has been reported that interventions such as pre-operative rehabilitation and pre-operative education are effective in improving post-operative physical and mental functions in CABG and valve surgery [34-36]. However, because aortic surgery often involves emergency surgery associated with aneurysm rupture or dissection and because there is a risk of rupture even in cases that are asymptomatic and discovered incidentally [37], it is often impossible to take measures such as rehabilitation intervention pre-operatively. Therefore, it is difficult for $\mathrm{CR}$ in the aortic surgery group to 
achieve similar physical recovery with the same interventions as the other two surgeries, suggesting the need for more extensive post-operative interventions.

The present study has several limitations. First, our study was retrospective and conducted at a single center. Thus, the external validity was insufficient. There may be some bias in the progression of $\mathrm{CR}$, and larger multicenter studies may provide additional insight into $\mathrm{CR}$ after cardiovascular surgery. Second, the pre-operative physical function was not assessed in this study; therefore, the association of progress of $\mathrm{CR}$ with prior physical function could not be studied. Future research will be needed to evaluate pre-and post-operative physical function.

A strength of this study was that it included patients with more extended demographics compared with previous studies; we identified multiple factors that contributed to delayed independent walking after cardiovascular surgery, including aortic surgery.

\section{Conclusions}

Independent walking is a fundamental activity after discharge from cardiovascular surgery to prevent a recurrence, to continue exercise, and to live an independent daily life. In this study, we showed that there are factors common to the three surgical groups and factors specific to each surgical group that determine post-operative independent walking. In particular, aortic surgery showed delayed post-operative recovery compared with the other two surgical groups, suggesting the need for a more generous support and approach than conventional CR. Further studies are needed to determine how to tailor interventions to the characteristics of each cardiovascular surgery.

Author Contributions: Conceptualization, Y.S. and R.K.; methodology, Y.S. and R.K.; formal analysis, Y.S. and R.K.; investigation, Y.S., Y.M., M.H., Y.Y., T.S., T.M., K.E. and R.K.; resources, Y.S.; writingoriginal draft preparation, Y.S.; writing-review and editing, Y.S., Y.M., M.H. and R.K. All authors have read and agreed to the published version of the manuscript.

Funding: This research received no external funding.

Institutional Review Board Statement: The study was conducted according to the guidelines of the Declaration of Helsinki and approved by the Nagasaki University Hospital Clinical Research Ethical Committee (reference number 15061113-2, 11 January 2017).

Informed Consent Statement: Informed consent was obtained from all subjects involved in the study in the form of an opt-out option on the website; those who were not interested were excluded.

Data Availability Statement: The data that support the findings of this study are available from the corresponding author upon reasonable request.

Acknowledgments: We acknowledge all of the staff members of the Department of Cardiopulmonary Rehabilitation Sciences, Nagasaki University Graduate School of Biomedical Sciences, and of the Department of Rehabilitation Medicine, Nagasaki University Hospital.

Conflicts of Interest: The authors declare no conflict of interest.

\section{References}

1. JCS Joint Working Group Guidelines for Rehabilitation in Patients With Cardiovascular Disease (JCS 2012). Circ. J. 2014, 78, 2022-2093. [CrossRef]

2. Cui, Z.; Li, N.; Gao, C.; Fan, Y.; Zhuang, X.; Liu, J.; Zhang, J.; Tan, Q. Precision implementation of early ambulation in elderly patients undergoing off-pump coronary artery bypass graft surgery: A randomized-controlled clinical trial. BMC Geriatr. 2020, 1, 1-10. [CrossRef]

3. Ohbe, H.; Nakamura, K.; Uda, K.; Matsui, H.; Yasunaga, H. Effect of Early Rehabilitation on Physical Function in Patients Undergoing Coronary Artery Bypass Grafting: A Nationwide Inpatient Database Study. J. Clin. Med. 2021, 10, 618. [CrossRef]

4. Dong, Z.; Yu, B.; Zhang, Q.; Pei, H.; Xing, J.; Fang, W.; Sun, Y.; Song, Z. Early Rehabilitation Therapy Is Beneficial for Patients With Prolonged Mechanical Ventilation After Coronary Artery Bypass Surgery. Int. Heart J. 2016, 57, 241-246. [CrossRef]

5. Ibrahim, K.; McCarthy, C.P.; McCarthy, K.J.; Brown, C.H.; Needham, D.M.; Januzzi Jr, J.L.; McEvoy, J.W. Delirium in the Cardiac Intensive Care Unit. J. Am. Heart Assoc. 2018, 7, 1-11. [CrossRef]

6. Shirvani, F.; Naji, S.A.; Davari, E.; Sedighi, M. Early mobilization reduces delirium after coronary artery bypass graft surgery. Asian Cardiovasc. Thorac. Ann. 2020, 28, 566-571. [CrossRef] 
7. Balady, G.J.; Ades, P.A.; Bittner, V.A.; Franklin, B.A.; Gordon, N.F.; Thomas, R.J.; Tomaselli, G.F.; Yancy, C.W. Referral, enrollment, and delivery of cardiac rehabilitation/secondary prevention programs at clinical centers and beyond: A presidential advisory from the American Heart Association. Circulation 2011, 124, 2951-2960. [CrossRef]

8. Chaddha, A.; Eagle, K.A.; Braverman, A.C.; Kline-Rogers, E.; Hirsch, A.T.; Brook, R.; Jackson, E.A.; Woznicki, E.M.; HousholderHughes, S.; Pitler, L.; et al. Exercise and Physical Activity for the Post-Aortic Dissection Patient: The Clinician's Conundrum. Clin. Cardiol. 2015, 38, 647-651. [CrossRef] [PubMed]

9. Dylewicz, P.; Bienkowska, S.; Szczesniak, L.; Rychlewski, T.; Przywarska, I.; Wilk, M.; Jastrzebski, A. Beneficial effect of short-term endurance training on glucose metabolism during rehabilitation after coronary bypass surgery. Chest 2000, 117, 47-51. [CrossRef] [PubMed]

10. Hedbäck, B.E.; Perk, J.; Engvall, J.; Areskog, N.H. Cardiac rehabilitation after coronary artery bypass grafting: Effects on exercise performance and risk factors. Arch. Phys. Med. Rehabil. 1990, 71, 1069-1073.

11. Engblom, E.; Korpilahti, K.; Hämäläinen, H.; Rönnemaa, T.; Puukka, P. Quality of life and return to work 5 years after coronary artery bypass surgery. Long-term results of cardiac rehabilitation. J. Cardiopulm. Rehabil. 1997, 17, 29-36. [CrossRef] [PubMed]

12. JCS/JSCVS/JATS/JSVS 2020 Guideline on Diagnosis and Treatment of Aortic Aneurysm and Aortic Dissection. Circ. J. 2020. Available online: https:/ / www.j-circ.or.jp/cms /wp-content/uploads/2020/07/JCS2020_Ogino.pdf (accessed on 20 October 2021). (In Japanese).

13. Chaddha, A.; Kline-Rogers, E.; Woznicki, E.M.; Brook, R.; Housholder-Hughes, S.; Braverman, A.C.; Pitler, L.; Hirsch, A.T.; Eagle, K.A. Activity recommendations for postaortic dissection patients. Circulation 2014, 130, e140-e142. [CrossRef] [PubMed]

14. Franklin, B.A.; Brinks, J.; Sacks, R.; Trivax, J.; Friedman, H. Reduced walking speed and distance as harbingers of the approaching grim reaper. Am. J. Cardiol. 2015, 116, 313-317. [CrossRef] [PubMed]

15. Lo, A.X.; Donnelly, J.P.; McGwin, G., Jr.; Bittner, V.; Ahmed, A.; Brown, C.J. Impact of gait speed and instrumental activities of daily living on all-cause mortality in adults $\geq 65$ years with heart failure. Am. J. Cardiol. 2015, 115, 797-801. [CrossRef]

16. Saitoh, M.; Takahashi, T.; Sakurada, K.; Kumamaru, M.; Hanafusa, Y.; Iwatsu, K.; Tahara, M.; Oura, K.; Yuguchi, S.; Morisawa, T. Factors determining achievement of early postoperative cardiac rehabilitation goal in patients with or without preoperative kidney dysfunction undergoing isolated cardiac surgery. J. Cardiol. 2013, 61, 299-303. [CrossRef]

17. Kato, M.; Saitoh, M.; Kawamura, T.; Iwata, K.; Sakurada, K.; Okamura, D.; Tahara, M.; Yuguchi, S.; Kamisaka, K.; Oura, K.; et al. Postoperative atrial fibrillation is associated with delayed early rehabilitation after heart valve surgery: A multicenter study. Phys. Ther. Res. 2019, 22, 1-8. [CrossRef]

18. Iida, Y.; Yamazaki, T.; Kawabe, T.; Usui, A.; Yamada, S. Postoperative muscle proteolysis affects systemic muscle weakness in patients undergoing cardiac surgery. Int. J. Cardiol. 2014, 172, 595-597. [CrossRef]

19. Hirsch, C.H.; Sommers, L.; Olsen, A.; Mullen, L.; Winograd, C.H. The natural history of functional morbidity in hospitalized older patients. J. Am. Geriatr. Soc. 1990, 38, 1296-1303. [CrossRef]

20. Monteleone, S.; Dalla Toffola, E.; Emiliani, V.; Ricotti, S.; Bruggi, M.; Conte, T.; D’Armini, A.M.; Orlandoni, G.; Petrucci, L. Recovery of deambulation after cardiothoracic surgery: A single center experience. Eur. J. Phys. Rehabil. Med. 2015, 51, 763-771.

21. Shimizu, H.; Okada, M.; Tangoku, A.; Doki, Y.; Endo, S.; Fukuda, H.; Hirata, Y.; Iwata, H.; Kobayashi, J.; Kumamaru, H.; et al. Thoracic and cardiovascular surgeries in Japan during 2017: Annual report by the Japanese Association for Thoracic Surgery. Gen. Thorac. Cardiovasc. Surg. 2020, 68, 414-449. [CrossRef]

22. Khan, F.M.; Naik, A.; Hameed, I.; Robinson, N.B.; Spadaccio, C.; Rahouma, M.; Yongle, R.; Demetres, M.; Chen, H.; Chang, M.; et al. Open Repair of Descending Thoracic and Thoracoabdominal Aortic Aneurysms: A Meta-Analysis. Ann. Thorac. Surg. 2020, 110, 1941-1949. [CrossRef]

23. Xu, S.; Liu, J.; Li, L.; Wu, Z.; Li, J.; Liu, Y.; Zhu, J.; Sun, L.; Guan, X.; Gong, M.; et al. Cardiopulmonary bypass time is an independent risk factor for acute kidney injury in emergent thoracic aortic surgery: A retrospective cohort study. J. Cardiothorac. Surg. 2019, 14, 90. [CrossRef] [PubMed]

24. Buth, J.; Harris, P.L.; Hobo, R.; van Eps, R.; Cuypers, P.; Duijm, L.; Tielbeek, X. Neurologic complications associated with endovascular repair of thoracic aortic pathology: Incidence and risk factors. a study from the European Collaborators on Stent/Graft Techniques for Aortic Aneurysm Repair (EUROSTAR) registry. J. Vasc. Surg. 2007, 46, 1103-1110; discussion 1110-1111. [CrossRef]

25. Chiesa, R.; Tshomba, Y.; Civilini, E.; Marone, E.M.; Bertoglio, L.; Baccellieri, D.; Coppi, G.; Logaldo, D.; Melissano, G. Open repair of descending thoracic aneurysms. HSR Proc. Intensive Care Cardiovasc. Anesth. 2010, 2, 177-190. [PubMed]

26. Takashima, N.; Ohkubo, T.; Miura, K.; Okamura, T.; Murakami, Y.; Fujiyoshi, A.; Nagasawa, S.Y.; Kadota, A.; Kita, Y.; Miyagawa, N.; et al. Long-term risk of BP values above normal for cardiovascular mortality: A 24-year observation of Japanese aged 30 to 92 years. J. Hypertens. 2012, 30, 2299-2306. [CrossRef] [PubMed]

27. Seino, Y.; Nanjo, K.; Tajima, N.; Kadowaki, T.; Kashiwagi, A.; Araki, E.; Ito, C.; Inagaki, N.; Iwamoto, Y.; Kasuga, M.; et al. Report of the committee on the classification and diagnostic criteria of diabetes mellitus. J. Diabetes Investig. 2010, 1, 212-228. [CrossRef]

28. Fliser, D.; Laville, M.; Covic, A.; Fouque, D.; Vanholder, R.; Juillard, L.; Van Biesen, W. A European Renal Best Practice (ERBP) position statement on the Kidney Disease Improving Global Outcomes (KDIGO) clinical practice guidelines on acute kidney injury: Part 1: Definitions, conservative management and contrast-induced nephropathy. Nephrol. Dial. Transplant. 2012, 27, 4263-4272. [CrossRef]

29. Edition, F. Diagnostic and statistical manual of mental disorders. Am. Psychiatric. Assoc. 2013, 21, 591-643. 
30. Canet, J.; Gallart, L.; Gomar, C.; Paluzie, G.; Valles, J.; Castillo, J.; Sabate, S.; Mazo, V.; Briones, Z.; Sanchis, J. Prediction of postoperative pulmonary complications in a population-based surgical cohort. Anesthesiology 2010, 113, 1338-1350. [CrossRef]

31. Bailey, M.L.; Richter, S.M.; Mullany, D.V.; Tesar, P.J.; Fraser, J.F. Risk factors and survival in patients with respiratory failure after cardiac operations. Ann. Thorac. Surg. 2011, 92, 1573-1579. [CrossRef]

32. Leditschke, I.A.; Green, M.; Irvine, J.; Bissett, B.; Mitchell, I.A. What are the barriers to mobilizing intensive care patients? Cardiopulm. Phys. Ther. J. 2012, 23, 26-29. [CrossRef] [PubMed]

33. Tabuchi, Y.; Morimoto, Y.; Yano, Y.; Tawara, Y.; Sato, S.; Tanigawa, K.; Eishi, K.; Kozu, R. Risk Factors for Post-operative Respiratory Failure and Respiratory Tract Infections Following Cardiovascular Surgery. Kyobu Geka 2017, 70, 1051-1062. (In Japanese) [PubMed]

34. Waite, I.; Deshpande, R.; Baghai, M.; Massey, T.; Wendler, O.; Greenwood, S. Home-based preoperative rehabilitation (prehab) to improve physical function and reduce hospital length of stay for frail patients undergoing coronary artery bypass graft and valve surgery. J. Cardiothorac. Surg. 2017, 12, 91. [CrossRef] [PubMed]

35. Steinmetz, C.; Bjarnason-Wehrens, B.; Baumgarten, H.; Walther, T.; Mengden, T.; Walther, C. Prehabilitation in patients awaiting elective coronary artery bypass graft surgery - effects on functional capacity and quality of life: A randomized controlled trial. Clin. Rehabil. 2020, 34, 1256-1267. [CrossRef]

36. Golaghaie, F.; Esmaeili-Kalantari, S.; Sarzaeem, M.; Rafiei, F. Adherence to lifestyle changes after coronary artery bypass graft: Outcome of preoperative peer education. Patient Educ. Couns. 2019, 102, 2231-2237. [CrossRef]

37. Erbel, R.; Aboyans, V.; Boileau, C.; Bossone, E.; Bartolomeo, R.D.; Eggebrecht, H.; Evangelista, A.; Falk, V.; Frank, H.; Gaemperli, O.; et al. 2014 ESC Guidelines on the diagnosis and treatment of aortic diseases: Document covering acute and chronic aortic diseases of the thoracic and abdominal aorta of the adult. The Task Force for the Diagnosis and Treatment of Aortic Diseases of the European Society of Cardiology (ESC). Eur. Heart J. 2014, 35, 2873-2926. [CrossRef] 